\title{
GIS-based expeditious assessments in the field of water protection
}

\author{
Francesco Mundo $^{\text {a, }}$, , Massimo Peleggi a , Silvana Salvati ${ }^{\text {a }}$ \\ a ISPRA (Italian Institute for Environmental Protection and Research), francesco.mundo@isprambiente.it, \\ massimo.peleggi@isprambiente.it, silvana.salvati@isprambiente.it \\ * Corresponding author
}

Keywords: Digital thematic map, Nitrates Vulnerable Zones, Nitrates hotspots, Agricultural pressure, Water protection

\begin{abstract}
:
The present abstract shows how expeditious GIS-based assessments can be used for a preliminary check of the correct implementation of certain measures aimed at the protection of water bodies from nutrient pollution and eutrophication pursuant to relevant EU legislation.
\end{abstract}

Two main pillars of the European legislation on water protection against enrichment with nutrients and potential eutrophication are Directive 91/676/EEC (Nitrates Directive or ND) and Directive 91/271/EEC (Urban Waste Water Treatment Directive or UWWTD). A common approach is established by these Directives, which requires the designation of specific "protected areas" (i.e. Nitrates Vulnerable Zones and Sensitive Areas respectively) on the territory, where relevant stringent measures apply (e.g. fertilization bans, nitrogen maximum application limits to soil, more stringent treatment for nitrogen and phosphorus removal in urban waste water treatment plants). The designation of Nitrates Vulnerable Zones (NVZs) is triggered by specific conditions set out in ND. One of the main criteria is that all known areas of land that drain into waters having nitrates $\left(\mathrm{NO}_{3}^{-}\right)$concentration higher than $50 \mathrm{mg} / \mathrm{l}$ and contribute significantly to such pollution must be designated as vulnerable zones.

Figure 1a below shows, just as an example, hotspots on the territory of Sardinia region, deriving from data submitted for the period 2016-2019 under Article 10 of ND. Notably, red dots (hotspots) are groundwater monitoring points with average $\mathrm{NO}_{3}^{-}$concentration above $50 \mathrm{mg} / \mathrm{l}$ outside $\mathrm{NVZs}$ designated until 2019. These points deserve particular attention since, if their pollution is caused, also only partly, by agricultural pressure, they need to be reclaimed through the measures of an action programme under ND, which applies inside NVZs. In Figure 1b, NVZs on the regional territory are shown, which have been designated after 2019, in order to include hotspots, which provides indication of potentially correct implementation of ND provisions concerning NVZs designation.

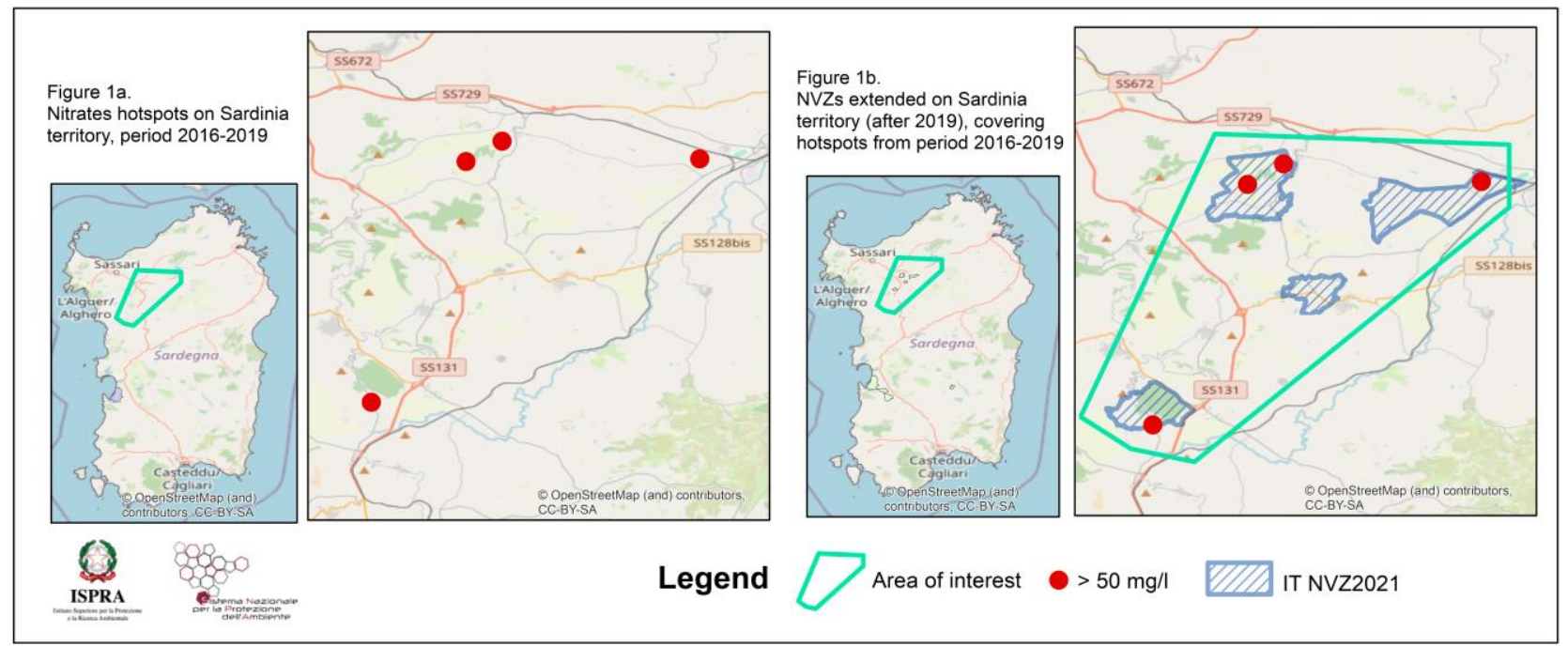

Figure 1. a. Examples of Nitrates hotspots on Sardinia territory, period 2016-2019; b. NVZs designated on Sardinia territory after 2019 and hotspots from period 2016-2019. Authors' elaboration on OpenStreetMap map (C) OpenStreetMap contributors, https://www.openstreetmap.org/copyright/en).

Figure 2 shows an example of designated NVZs on the territory of Puglia region, which cover an area with relatively high presence of livestock, thus indicating, also in this case, a potentially correct implementation of ND provisions concerning NVZs. 


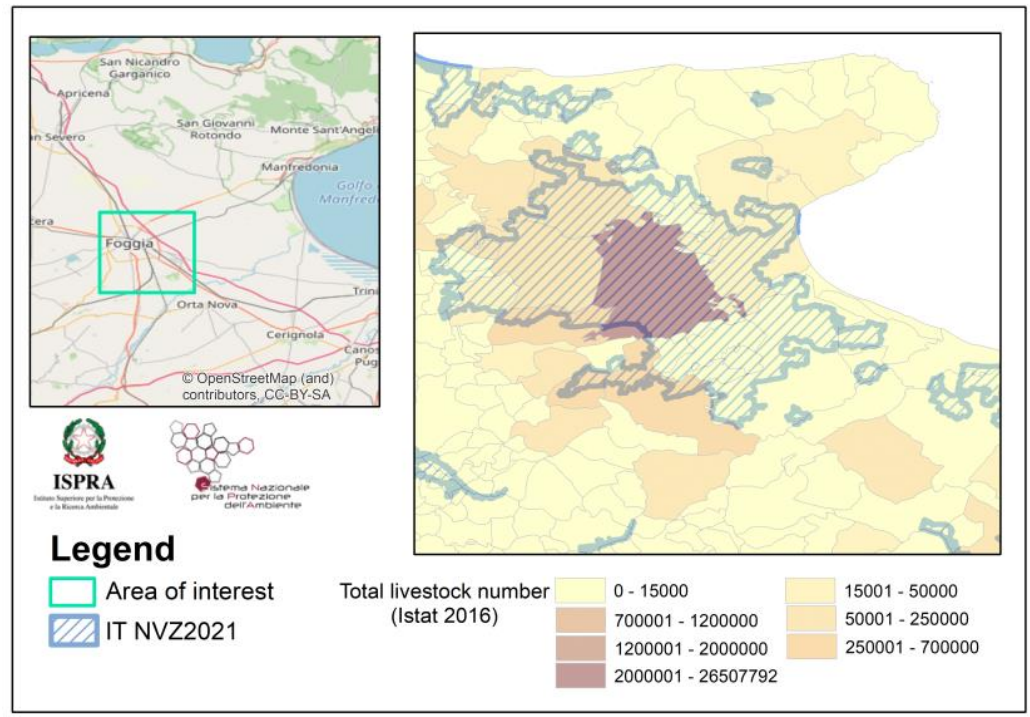

Left image - Authors' elaboration on

OpenStreetMap map

(@) OpenStreetMap contributors,

https://www.openstreetmap.org/copyright/en)

Right image - Authors' elaboration on

administrative boundaries layer by ISTAT

(Istituto Nazionale di Statistica -

https://www.istat.it/it/archivio/222527, License

http://creativecommons.org/licenses/by/3.0/it/)

Figure 2. Example of NVZ designated in Puglia region.

Even though, on the one hand, the designation or the extension of NVZs aimed at including hotspots can be seen as a sign of a correct response by competent authorities, on the other hand, the presence of hotspots outside NVZs does not necessarily imply a breach of the Directive. Nevertheless, these circumstances need a closer look in order to establish whether or not they were unduly neglected upon designation of NVZs. This assessment can be carried out by adding more layers, separately or altogether, to a digital thematic map (GIS), which contain data concerning, inter alia, livestock density, the presence of discharges from urban wastewater treatment plants, local geology, providing precious information respectively on the potential agricultural pressure, the presence of alternative $\mathrm{NO}_{3}^{-}$pollution sources and the intrinsic aquifer vulnerability in case the concentration limits have been exceeded in groundwater monitoring points. Furthermore, satellite or aerial imagery can be used for detecting the presence of specific features (e.g. non registered or unknown discharge points or wastewater treatment plants), which can explain the presence of unexpected hotspots or a DEM and hydrography layer, which can help establish, inter alia, most probable pollution pathways and single out potential pollution sources or exclude the presence of an agricultural pressure.

Figure $3(a, b, c)$ below shows examples of nitrates hotspots, which are most likely caused by pressures other than agriculture and, therefore, in principle, do not need to be included in NVZs.

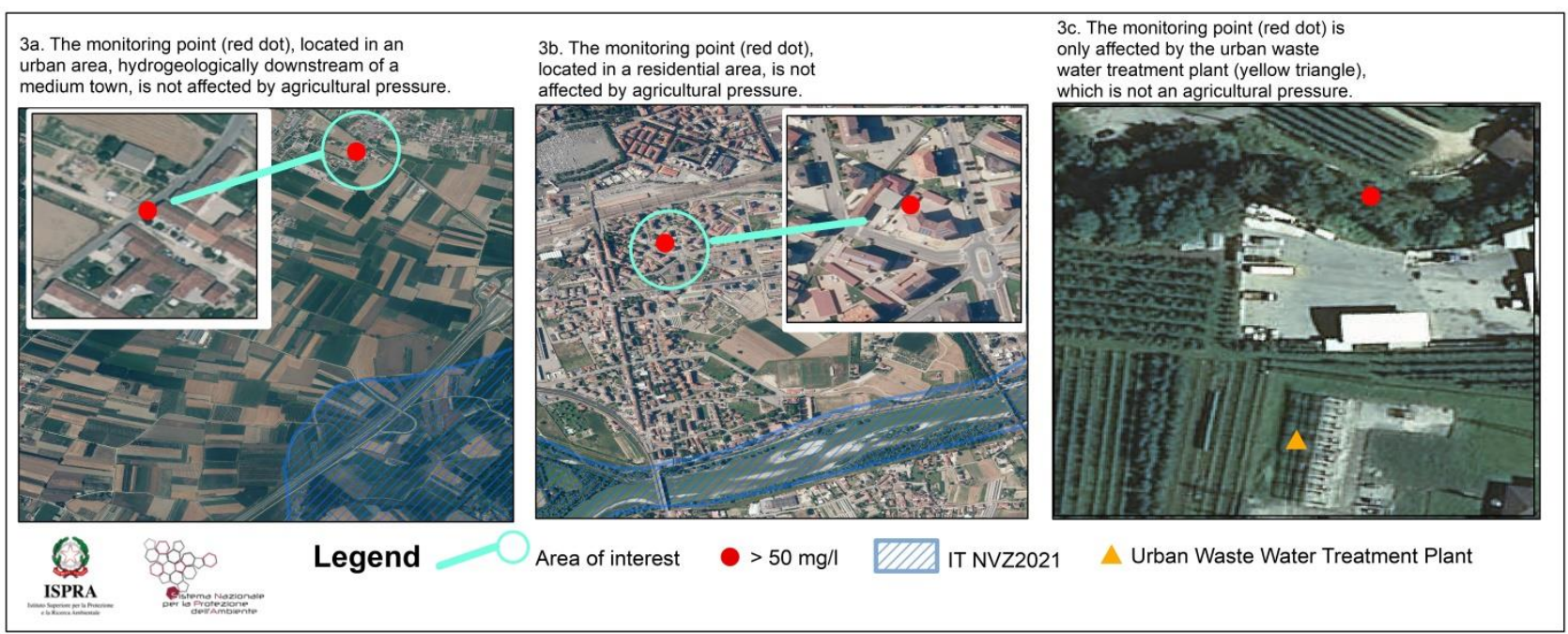

Figure 3. Examples of nitrates hotspots not linked to agricultural pressures (3a. Urban area; 3b. Residential area; 3c. Pressure from urban waste water treatment plant). Authors' elaboration on AGEA 2012 orthophoto layer by AGEA (Agenzia per le erogazioni in agricoltura, License http://creativecommons.org/licenses/by/3.0/it/).

This abstract provides examples on potential use of GIS for preliminary assessments of compliance with EU Directives in the field of water protection (notably ND). This type of assessment is not meant to be exhaustive or conclusive and cannot replace in-depth evaluations for specific situations on a local level but can be used as an effective tool to quickly spot potential criticalities concerning the implementation of Nitrates Directive, notably as regards the designation of NVZs. Based on these expeditious assessments, more in-depth scrutiny can be effectively designed and oriented towards a small set of unclear situations where additional information (on-spot surveys, inspections, studies, archive consultation, paperwork, etc.) is really needed, thus reducing the administrative burden on competent authorities while increasing the effectiveness and efficiency of their action.

Statement on competing interests: the authors declare that they have no conflict of interest. 